\title{
Thomson scattering on inhomogeneous targets
}

\author{
R. Thiele, ${ }^{1, *}$ P. Sperling, ${ }^{1}$ M. Chen, ${ }^{2,3}$ Th. Bornath, ${ }^{1}$ R. R. Fäustlin, ${ }^{4}$ C. Fortmann,,${ }^{5,6}$ S. H. Glenzer, ${ }^{6}$ W.-D. Kraeft, ${ }^{1}$ \\ A. Pukhov, ${ }^{3}$ S. Toleikis, ${ }^{4}$ Th. Tschentscher, ${ }^{7}$ and R. Redmer ${ }^{1}$ \\ ${ }^{1}$ Institut für Physik, Universität Rostock, D-18051 Rostock, Germany \\ ${ }^{2}$ Lawrence Berkeley National Laboratory, 1 Cyclotron Road, MS 71-259, Berkeley, California 94720, USA \\ ${ }^{3}$ Institut für Theoretische Physik I, Heinrich-Heine-Universität Düsseldorf, D-40225 Düsseldorf, Germany \\ ${ }^{4}$ DESY, Notkestraße 85, D-22607 Hamburg, Germany \\ ${ }^{5}$ Department of Physics and Astronomy, University of California-Los Angeles, Los Angeles, California 90095, USA \\ ${ }^{6}$ Lawrence Livermore National Laboratory, P.O. Box 808, L-399, Livermore, California 94551, USA \\ ${ }^{7}$ European XFEL GmbH, Albert-Einstein-Ring 19, D-22761 Hamburg, Germany
}

(Received 17 May 2010; published 4 November 2010)

\begin{abstract}
The introduction of brilliant free-electron lasers enables new pump-probe experiments to characterize warm dense matter states. For instance, a short-pulse optical laser irradiates a liquid hydrogen jet that is subsequently probed with brilliant soft $\mathrm{x}$-ray radiation. The strongly inhomogeneous plasma prepared by the optical laser is characterized with particle-in-cell simulations. The interaction of the soft $\mathrm{x}$-ray probe radiation for different time delays between pump and probe with the inhomogeneous plasma is also taken into account via radiative hydrodynamic simulations. We calculate the respective scattering spectrum based on the Born-Mermin approximation for the dynamic structure factor considering the full density and temperature-dependent Thomson scattering cross section throughout the target. We can identify plasmon modes that are generated in different target regions and monitor their temporal evolution. Therefore, such pump-probe experiments are promising tools not only to measure the important plasma parameters density and temperature but also to gain valuable information about their time-dependent profile through the target. The method described here can be applied to various pump-probe scenarios by combining optical lasers and soft $\mathrm{x}$ ray, as well as $\mathrm{x}$-ray sources.
\end{abstract}

DOI: 10.1103/PhysRevE.82.056404

PACS number(s): 52.25.Os, 52.35.Fp, 71.45.Gm, 71.10.Ca

\section{INTRODUCTION}

X-ray Thomson scattering has demonstrated its capacity as a reliable and versatile tool for the diagnostics of dense plasmas [1]. X rays emitted from laser produced plasmas $[2,3]$ can probe the warm dense matter region $[4,5]$ with temperatures of several $\mathrm{eV}$ and densities close to solid density [6-8] up to compressed matter well above solid density and electron temperatures $T_{e}$ between $0.1 \mathrm{eV}$ and several 10 $\mathrm{eV}$ [9-13]. These plasmas are opaque in the optical region since the frequency of light $\omega_{0}=2 \pi c / \lambda_{0}$ is lower than the plasma frequency $\omega_{p e}^{2}=n_{e} e^{2} /\left(\epsilon_{0} m_{e}\right)$ of the free-electron subsystem, with the free-electron density $n_{e}$ and the electron mass $m_{e}$. Therefore, probing plasmas with densities approaching solid or even higher densities requires efficient x-ray sources with high brightness, which can be produced by energetic optical lasers $[14,15]$. Alternatively, the study of dense plasmas is now possible with the implementation of free-electron lasers (FEL) which provide brilliant radiation in the soft X-ray region as at the Free Electron Laser Hamburg (FLASH) at DESY, Hamburg [16] or in the x-ray region as at the Linac Coherent Light Source (LCLS) in Stanford [17] and at the future European x-ray free electron laser (XFEL) in Hamburg [18].

Collective x-ray Thomson scattering experiments yield information on the density and temperature of dense plasmas [8]. Assuming homogeneous density and temperature throughout the target, these parameters can be determined

\footnotetext{
*robert.thiele@uni-rostock.de
}

directly from the plasmon dispersion and the ratio of the plasmon amplitudes via the detailed balance relation [19]. In optical laser-plasma interaction, the target is overdense and the absorption is limited to the skin depth, in contrast to excitation by short-wavelength FEL radiation where gradients are smaller (see $[20,21]$ ). The scattering signal represents an average of the local density- and temperaturedependent scattering cross sections weighted with the respective density and temperature profiles [22]. These profiles are generated not only by the pump pulse but also by the probe pulse if intense $\mathrm{x}$-ray radiation pulses are used as in the case of the FLASH and LCLS facilities. Therefore, a realistic description of the light-matter interaction within a pump-probe experiment is of paramount importance to derive reliable information on the plasma parameters, density and temperature, their profiles throughout the target, and their temporal evolution.

The prospects of brilliant $\mathrm{x}$-ray sources for probing soliddensity plasmas have been shown earlier via calculations and theoretical analysis. For instance, the evolution of an aluminum plasma has been determined on picosecond time scales within a pump (optical short-pulse laser with $\lambda_{0}=1 \mu \mathrm{m}$, $\tau=500 \mathrm{fs}$, and $E_{0}=1 \mathrm{~J}$ )-probe (soft x-ray source with $\lambda_{0}=14.7 \mathrm{~nm}, \tau=5 \mathrm{ps}$, and $\left.E_{0}=0.5 \mathrm{~mJ}\right)$ scenario by using standard expressions for the dynamic structure factor (DSF) for collisionless plasmas [22].

Self-Thomson scattering off liquid hydrogen targets, i.e., generating and probing the plasma simultaneously with only one ultrashort soft $\mathrm{x}$-ray pulse, has been studied for conditions at FLASH $\left(\lambda_{0}=13.5 \mathrm{~nm}, \tau=30 \mathrm{fs}\right.$, and $\left.E_{0}=0.05 \mathrm{~mJ}\right)$ [23]. Collisions in the plasma were considered within the Born-Mermin approximation (BMA); only moderate density 
and temperature gradients were imposed by the FLASH laser due to the rather large absorption length of $11 \mu \mathrm{m}$ for this wavelength [24].

In this paper we consider the interaction of both short $800 \mathrm{~nm}$ optical and $13.5 \mathrm{~nm}$ soft $\mathrm{x}$-ray pulses with a liquid hydrogen target within a full pump-probe scenario as performed at FLASH $[19,23]$ with time delays of 1-10 ps. We apply particle-in-cell (PIC) as well as radiationhydrodynamic codes for the laser-target interaction and consider collisions in the plasma via the BMA when calculating the DSF. Our results show that not only the important plasma parameters density and temperature but also information about their time-dependent profile through the target can be derived from the Thomson scattering signal. This theoretical analysis is of basic interest for future experiments at FLASH and LCLS.

Our paper is organized as follows. We define in Sec. II the Thomson scattering power via the DSF $S_{e e}(k, \omega)$ [25] that is a function of the scattering wave number $k$ and the frequency shift $\omega$, integrated with the density and temperature distribution of the target. The DSF is determined in the BMA for the dielectric function $\epsilon(k, \omega)$. The influence of plasma inhomogeneities in the target on the Thomson scattering signal is studied in Sec. III, and conclusions are given in Sec. IV.

\section{THEORY OF THOMSON SCATTERING}

\section{A. Dynamic structure factor}

We start with the experimentally available scattering power per solid angle $d \Omega=\sin \vartheta d \vartheta d \varphi$ and per unit frequency interval $d \omega$, which is described by the following expression [22]:

$$
\begin{aligned}
\frac{d^{2} P_{s c}}{d \Omega d \omega}= & \frac{\sigma_{T}}{A_{r a d}} \frac{k_{f}}{k_{i}} \int_{-\infty}^{\infty} \frac{d \omega^{\prime}}{2 \pi} G_{\Delta \omega}\left(\omega-\omega^{\prime}\right) \\
& \times \int d^{3} \mathbf{r} l(\mathbf{r}) S_{e e}\left[\mathbf{k}, \omega^{\prime} ; n_{e}(\mathbf{r}), T_{e}(\mathbf{r})\right] n_{i}(\mathbf{r}) .
\end{aligned}
$$

Here, $\sigma_{T}=6.65 \times 10^{-24} \mathrm{~cm}^{2}$ is the Thomson scattering cross section, $k_{i}$ and $k_{f}$ are the initial and final photon wave numbers, and the energy and momentum transfer are given by $\Delta E=\hbar \omega=\hbar \omega_{f}-\hbar \omega_{i}$ and $\hbar \mathbf{k}=\hbar \mathbf{k}_{f}-\hbar \mathbf{k}_{i}$. The momentum is related to the scattering angle $\theta$ in the limit $\hbar \omega \ll \hbar \omega_{0}$ according to $k=4 \pi \sin (\theta / 2) / \lambda_{0}$, with $\lambda_{0}$ being the probe wavelength. $l(\mathbf{r})$ is the $\mathbf{r}$-dependent power density of incoming photons which is also dependent on the absorption, $n_{i}(\mathbf{r})$ is the ion density, and $A_{\text {rad }}$ is the irradiated surface of the target. The DSF has to be convoluted with the instrumental function $G_{\Delta \omega}(\omega)$ that models the spectrometer's finite spectral resolution as well as the probe's spectral bandwidth. Usually, a normalized Gaussian distribution is employed with the full width at half maximum $\Delta \omega$. In this paper, the instrumental function is not considered. Furthermore, the DSF depends on the profiles of electron density $n_{e}(\mathbf{r})$ and temperature $T_{e}(\mathbf{r})$ throughout the target.
We follow Chihara's approach to the DSF $[25,26]$,

$$
\begin{aligned}
S_{e e}(k, \omega)= & Z_{f} S_{e e}^{0}(k, \omega)+\left|f_{i}(k)+q(k)\right|^{2} S_{i i}(k, \omega) \\
& +Z_{c} \int_{-\infty}^{\infty} d \omega^{\prime} S_{c}(k, \omega) S_{s}\left(k, \omega-\omega^{\prime}\right),
\end{aligned}
$$

which is decomposed into contributions of free electrons, weakly and tightly bound electrons, and core electrons. In this paper, the DSF of free electrons is considered, i.e., the first term. The calculation of this part can be found in Sec. II B. The second part of Eq. (2) gives us the correlation of the weakly and bound electrons following the ion motion. The amplitude is determined by the atomic form factor $f_{i}(k)$ and the screening cloud $q(k)$ of quasi-free-electrons which screen the ion charge [27].

The third term contains the contribution of core electrons via $S_{c}(k, \omega)$ and describes Raman-type transitions of inner shell electrons to the continuum, modulated by the ion movement which is contained in $S_{s}(k, \omega)$ [28]. The last term in Eq. (2) can be neglected for the hydrogen plasma studied here [19].

The scattering wave number $k$ is fixed through the geometry of the experimental setup, so that a scattering parameter $\alpha=\kappa / k$ can be introduced [29] that allows us to discriminate between collective $(\alpha>1)$ and noncollective $(\alpha<1)$ scattering; $\kappa$ is the density- and temperature-dependent screening parameter (see [1] for details). For $\alpha<1$, we can investigate short-range correlations within the Debye sphere [15], while long-range correlations are relevant for collective scattering, i.e., $\alpha>1$.

In the latter case, the DSF exhibits two pronounced side maxima, symmetrically redshifted and blueshifted with respect to the quasielastic Rayleigh peak which is related to the second term in Eq. (2). These features are collective electron plasma oscillations (plasmons) [8]. To first order, their position is given by the electron plasma frequency $\omega_{p e}$ which allows us to determine the electron density $n_{e}$. Thermal pressure corrections and quantum diffraction effects lead to a $k$ dependence in the plasmon dispersion (see, e.g., [30]).

\section{B. Born-Mermin approximation}

We focus on the first term in Eq. (2), the free-electron part. In thermodynamic equilibrium, $S_{e e}^{0}(k, \omega)$ is related to the longitudinal dielectric function $\epsilon(k, \omega)$ via the fluctuation-dissipation theorem

$$
S_{e e}^{0}(k, \omega)=-\frac{\epsilon_{0} \hbar k^{2}}{\pi e^{2} n_{e}} \frac{\operatorname{Im} \epsilon^{-1}(k, \omega)}{1-\exp \left(-\frac{\hbar \omega}{k_{B} T_{e}}\right)} .
$$

Neglecting collisions as in Ref. [22], the dielectric function is given by the well-known random-phase approximation (RPA); see [1,19] for details.

To go beyond the RPA, we follow Mermin's approach [31] which considers collisions within a Drude-like ansatz with a constant collision frequency $\nu$ for the damping of the frequency-dependent dielectric function. Applying linearresponse theory, the Mermin dielectric function can be gen- 
eralized to include a dynamic collision frequency $\nu(\omega)$ according to [32]

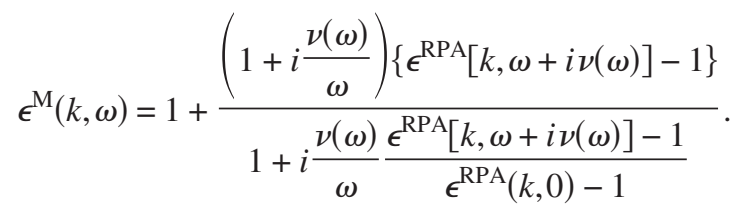

We evaluate the collision frequency in Born approximation with respect to a statically screened (Debye) potential $V_{D}(q)=-Z e^{2} /\left[\epsilon_{0}\left(q^{2}+\kappa_{D}^{2}\right)\right][19,33,34]$

$$
\begin{aligned}
\nu^{\mathrm{Born}}(\omega)= & -i \frac{\epsilon_{0} n_{i}}{6 \pi^{2} e^{2} n_{e} m_{e}} \frac{1}{\omega} \int_{0}^{\infty} d q q^{6} V_{D}^{2}(q) S_{i}(q) \\
& \times\left[\epsilon^{\mathrm{RPA}}(q, \omega)-\epsilon^{\mathrm{RPA}}(q, 0)\right] .
\end{aligned}
$$

Here, $S_{i}(q)$ is the static ion-ion structure factor and $\kappa_{D}$ $=\sqrt{n_{e} e^{2} /\left(\epsilon_{0} k_{B} T_{e}\right)}$ is the inverse (Debye) screening length.

The influence of collisions has been discussed in several papers (see [35-37]). Here, we calculate $S_{e e}^{0}(k, \omega)$ in BMA and focus on the impact of density and temperature profiles on the scattering signal.

\section{PUMP-PROBE SCENARIO}

In this section, we describe the interaction of an optical pump laser with a cold hydrogen target by means of largescale PIC simulations, and the interaction of this strongly inhomogeneous plasma with the soft $\mathrm{x}$-ray probe radiation via radiative hydrodynamic simulations. We calculate the resulting Thomson scattering spectrum and derive the respective plasma parameters.

\section{A. Density and temperature profiles impressed by a short-pulse optical laser}

The electron-density and temperature profiles in spherical hydrogen droplets are obtained from three-dimensional PIC simulations using the code VLPL3D [38] including fully relativistic ionization [39]. The linearly polarized laser pulse of $\lambda_{0}=800 \mathrm{~nm}$ wavelength, $t=150 \mathrm{fs}$ duration, and $E$ $=1.6 \mathrm{~mJ}$ energy is incident from the left with a focal radius of $r_{F}=20 \mu \mathrm{m}$ (see Fig. 1). These parameters that are available at FLASH imply that the electron dynamics and collisions are dominated by the strong laser field. Here, we used a collisionless version of the code and assumed field ionization. The cubic simulation box has a box length of $33.6 \mu \mathrm{m}$ and is divided into $2100(x) \times 140(y) \times 140(z)$ cells. We use 252 processors to perform the calculations, which is close to the upper limit of our current computational capacity. The initial density of the spherical liquid hydrogen target with $r_{H}=15 \mu \mathrm{m}$ is $\rho_{H}=0.086 \mathrm{~g} / \mathrm{cm}^{3}$ as used in experiment [23]. The target is initially located in the center of the simulation box. The computer capacity currently available allows us to run the code for a simulation time of 1 ps.

The resulting spatial electron-density and temperature distributions in the $x-y$ plane are shown in Figs. 2 and 3. Here, we use now a $50(x) \times 10(y)$ grid for the evaluation of the electron temperature (see Fig. 1). The cells $(0.67$

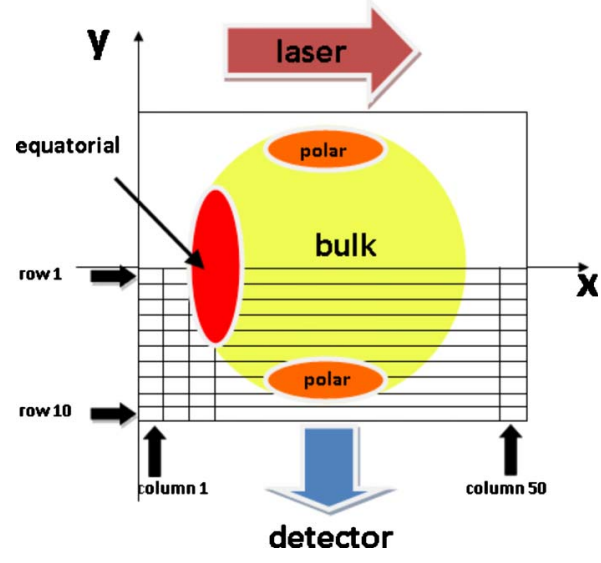

FIG. 1. (Color online) An optical laser irradiates liquid hydrogen droplets from left. The mesh for the PIC simulation (columns and rows) and regions within the droplet (equatorial, polar, and bulk) are defined; see also Secs. III B and III D.

$\times 1.68 \mu \mathrm{m}^{2}$ ) in the $z$ direction are integrated for each cell in the $x-y$ plane. Due to the symmetry, only half of the target is shown.

The PIC simulations indicate that the laser generates a strongly inhomogeneous plasma in the droplet with high ionization in a thin layer of $5 \lambda_{0} \approx 4 \mu \mathrm{m}$ and weak ionization in the bulk. The maximum free-electron density is induced in the equatorial region with values up to about $n_{\max }=100 n_{c r}$ $\approx 10^{23} \mathrm{~cm}^{-3}$. The free-electron densities in the polar regions of the droplet are still well above the critical density, while the bulk region is only weakly ionized and rather cold. The critical density is defined as

$$
n_{c r}=\frac{4 \pi^{2} c^{2}}{\lambda_{0}^{2}} \frac{\epsilon_{0} m_{e}}{e^{2}} .
$$

For a wavelength of $\lambda_{0}=800 \mathrm{~nm}$, it amounts to $n_{c r}=1.7$ $\times 10^{21} \mathrm{~cm}^{-3}$. The optical laser heats the equatorial region strongest (see Fig. 3), and the maximum electron temperature is about $9 \mathrm{eV}$. Only a few superthermal electrons accelerated by the laser pulse have been transported into the target. Behind the thin high-density layer of about four to five cells thickness $(\approx 4 \mu \mathrm{m})$, the density decreases rapidly by three orders of magnitude and drops only slightly in the following region up to the rear side of the droplet. The temperature decreases from about 9 to $1 \mathrm{eV}$ less strongly through the droplet.

\section{B. "Ideal" probe Thomson scattering}

In the next step, we calculate the DSF $S_{e e}^{0}(k, \omega) \omega_{p e}^{2}$ [see Eqs. (1) and (2)] for a scattering angle of $\theta_{S}=90^{\circ}$ for each cell with the free-electron densities and temperatures as obtained from the PIC simulations, altogether 336 cells with densities above $10^{18} \mathrm{~cm}^{-3}$. Each cell is weighted by the factor $\exp \left(-r / l_{a b s}\right)$, with the global absorption length of $l_{a b s}$ $=11 \mu \mathrm{m}$ that was used also in [24]. In this way we consider the weakening of the incoming FLASH probe pulse with increasing target depth. The internal feedback of the soft $\mathrm{x}$-ray photons onto the preheated target however is neglected 


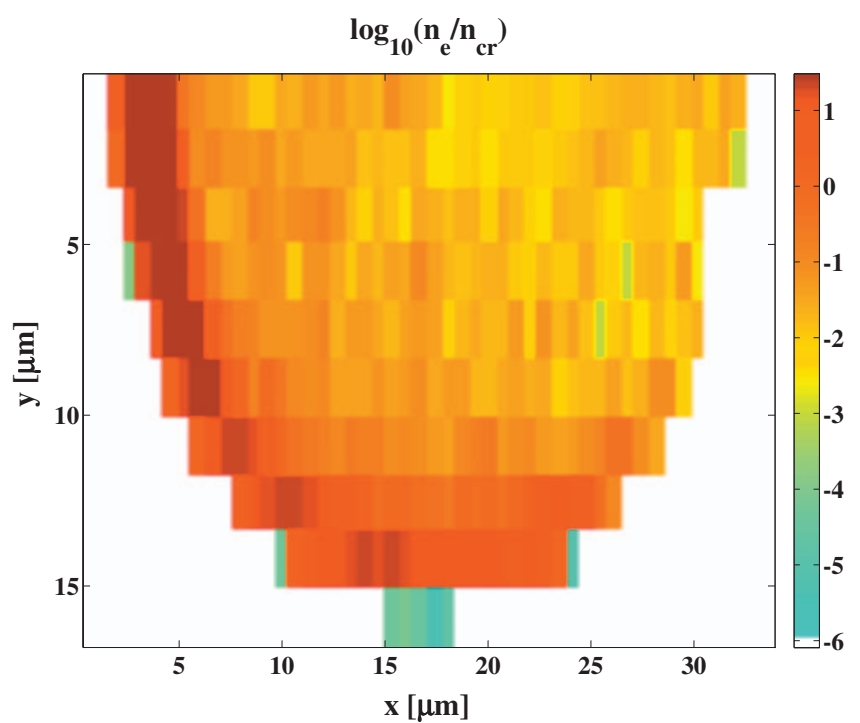

FIG. 2. (Color) Free-electron-density profile $n_{e}(x, y)$ in units of $n_{c r}$ (logarithmic scale, color coded) as derived from the PIC simulations; see also Sec. III A. The linearly polarized laser pulse of 800 $\mathrm{nm}$ wavelength and $150 \mathrm{fs}$ pulse length is incident from the left.

which would lead to further ionization and heating (see Sec. III C). Therefore, we denote this process as ideal probe. The parameters of the FLASH pulse are $\lambda_{0}=13.5 \mathrm{~nm}, \tau=30 \mathrm{fs}$, and $E_{0}=0.05 \mathrm{~mJ}$ (see [24]).

In Fig. 4, we show the integrated Thomson scattering signal as a function of the photon energy shift $\Delta E$ with respect to the FLASH photon energy of $E=92 \mathrm{eV}$ for two typical rows, i.e., integrating the contributions of all cells in that row (see Fig. 1). The calculation for row 1 (solid line) is typical for the central part of the droplet, i.e., rows 1-6. The peaks stem from two different scattering regions in the droplet. Collective scattering $(\alpha>1)$ is relevant for the high plasma densities in the equatorial region that yields the asymmetric plasmon feature. The remaining contributions are due to noncollective scattering from the cells with lower densities,

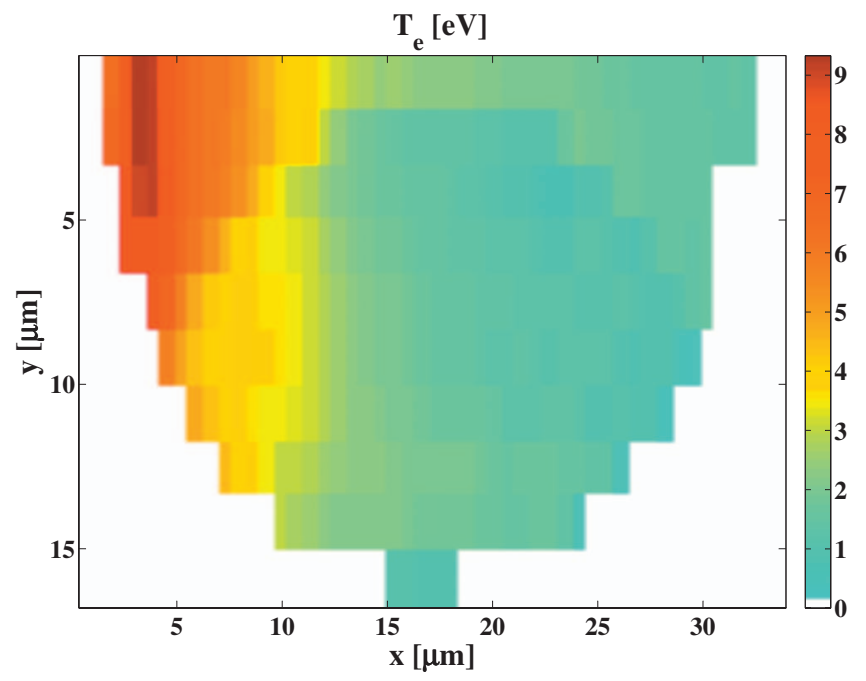

FIG. 3. (Color) Temperature profile of free electrons $T_{e}(r)$ as derived from the PIC simulations (see also Fig. 2).

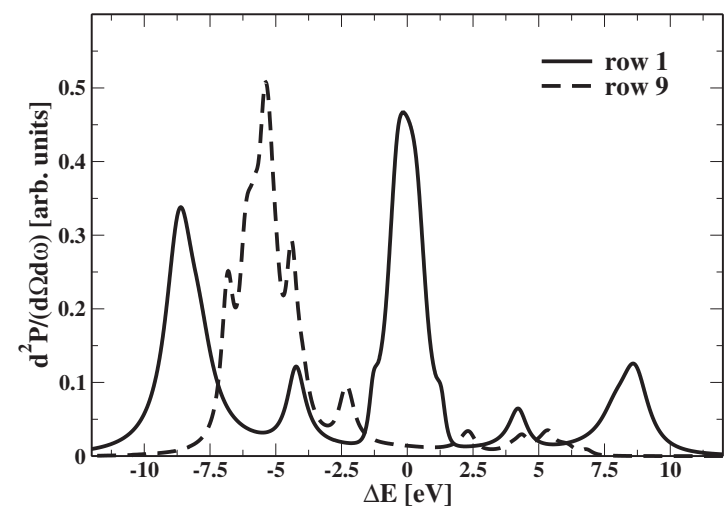

FIG. 4. Integrated Thomson scattering spectrum of a liquid hydrogen droplet for row 1 (solid line) and row 9 (broken line) as a function of the photon energy shift $\Delta E$ (see Fig. 1).

which leads to the central peak. Row 9 (broken line) is exemplary for rows 7-9 in the polar region and shows a strong collective scattering signal. The electron densities and temperatures are nearly constant or decrease only slowly in these rows (see also Figs. 2 and 3).

The contributions of each row and the resulting total scattering signal are shown in Fig. 5 as functions of the photon energy shift $\Delta E$. The high-energy peaks at $|\Delta E|=8.5 \mathrm{eV}$ stem from the equatorial region of the droplet. We derive an effective electron density of $n_{e}=5.3 \times 10^{22} \mathrm{~cm}^{-3}$ from the plasmon resonance and an effective temperature of $T_{e}$ $=7.6 \mathrm{eV}$ from the detailed balance relation; the respective mean temperature, the averaging of the corresponding cells of the equatorial region, $\bar{T}_{e} \approx 8.3 \mathrm{eV}$, differs by less than $10 \%$ from this effective value. The second plasmon peaks at $|\Delta E|=5.4 \mathrm{eV}$ are related to collective scattering from cells in the polar region of the droplet; an effective electron density of $n_{e}=2.1 \times 10^{22} \mathrm{~cm}^{-3}$ and an effective electron temperature of $T_{e}=3.9 \mathrm{eV}$ are found. The remaining bulk part of the droplet with a mean electron density of $\bar{n}_{e} \approx 5 \times 10^{19} \mathrm{~cm}^{-3}$ and an electron temperature of $\bar{T}_{e} \approx 2 \mathrm{eV}$ yields the central peak in Fig. 5, which is due to noncollective scattering events. The detailed balance and the dispersion relation are

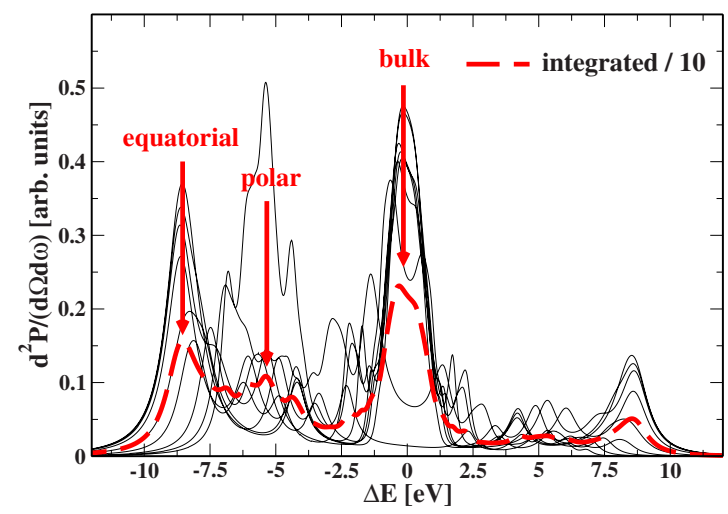

FIG. 5. (Color online) Total Thomson scattering spectrum divided by 10 (dashed red line) for the whole liquid hydrogen droplet as a function of the photon energy shift $\Delta E$ in comparison with the contributions of each row (black lines). 
not suitable here. Note that this is not the Rayleigh peak because elastic scattering off strongly bound electrons as described by the second term in Eq. (2) is not evaluated here.

\section{Absorption of the soft $x$-ray probe photons}

So far, we have calculated the spectrum of FLASH photons scattered off the inhomogeneous hydrogen droplet that was prepared by the optical laser. Absorption processes of FLASH photons in addition to Thomson scattering were neglected, so that Fig. 5 represents an ideal spectrum. We have treated self-Thomson scattering of FLASH photons in an earlier work, i.e., a restricted process without pumping the target with an optical laser but considering the full interaction of the soft x-ray photons with the target via radiationhydrodynamic simulations [24]. Moderate density and temperature gradients have been observed in the hydrogen droplet, in contrast to the present case where the optical laser generates strong gradients. The respective averaged spectrum has lead to effective values of temperature and density that were comparable with the averaged properties of the droplet.

We consider now the full pump-probe scenario by accounting for various absorption mechanisms in the target besides Thomson scattering. The PIC code cannot be applied for the probe pulse since the wavelength and sample size differ by many orders of magnitude. About $2 \times 10^{5}$ more simulation cells would be required compared to the optical laser case-well beyond the available computing capacity. Thus, we use instead the one-dimensional radiative hydrodynamic code HELIOS [40] for the interaction of the FLASH pulse $\left(\lambda_{0}=13.5 \mathrm{~nm}, \tau=30 \mathrm{fs}\right.$, and $\left.E_{0}=0.05 \mathrm{~mJ}\right)$ with the inhomogeneous hydrogen plasma. This code contains a Lagrangian reference frame, separated ion and electron temperatures, and flux-limited Spitzer thermal conductivity. Laser energy is deposited via inverse bremsstrahlung as well as bound-bound and bound-free transitions using a SESAMElike equation of state.

To simulate, in addition, time scales larger than $1 \mathrm{ps}$, we transfer the results of the PIC simulation to the radiative hydrodynamics code HELIOS. The sample is in a nonequilibrium state at the maximum PIC simulation time $(1 \mathrm{ps}$; see Figs. 2 and 3). However, HELIOS assumes start conditions in local thermal equilibrium (LTE). To bridge this gap, we calculate the sample's LTE temperature from the free-electron density after 1 ps (PIC results) using Saha equations [41,42]. The resulting approximation to the LTE temperature is used as a start condition for the HELIOS simulations. Uncertainties in the sample temperature become relatively large, and the LTE temperature deviates from the PIC results up to a factor of 3 in the polar region. However, this procedure allows us to span the femtosecond (PIC) to nanosecond (HELIOS) time scales while maintaining fairly high accuracy in terms of electron density. The sample evolution is simulated using HELIOS from 1 to $10 \mathrm{ps}$ after heating with the optical laser. At fixed time delays of $1,7.5$, and $10 \mathrm{ps}$, the FEL probe pulse is impinging on the sample, and its interaction is modeled using HELIOS. The FEL irradiation increases the free-electron density by up to two orders of magnitude in the sample's bulk region. At the same time, the density increases only

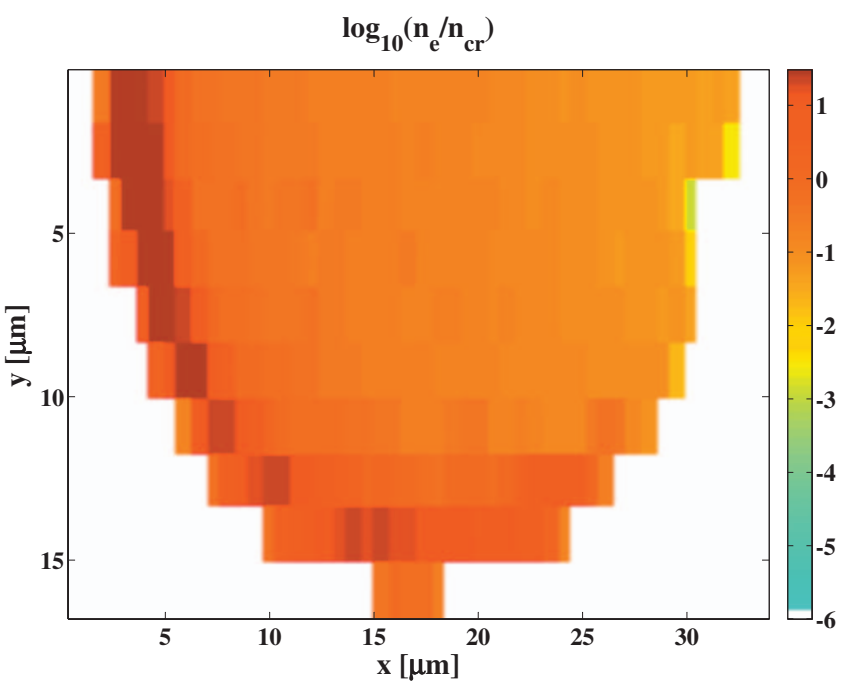

FIG. 6. (Color) Density of free electrons $n_{e}(x, y)$ in units of the critical density $n_{c r}$ of the optical laser (logarithmic scale, color coded) as derived from the HELIOS simulations. The FLASH pulse irradiates the droplet from the left 10 ps after the optical laser; see also Sec. III C.

marginally in the equatorial and polar regions which are already close to full ionization (see Fig. 6). Note that the FEL radiation penetrates deeply into the sample since the plasma density on the surface is well below the critical density for this short-wavelength radiation. Overall, the sample average temperature increases by about $10 \%$ due to the FEL probe irradiation.

The next step is the calculation of the scattering spectrum for several time delays $(1,7.5$, and $10 \mathrm{ps})$ for which we use now the sample density as derived from the HELIOS simulation. To minimize uncertainties from the PIC to HELIOS conversion, we use the relative temperature effect of the FEL radiation, i.e., we use the PIC temperature profile at $1 \mathrm{ps}$ increased by $10 \%$ due to FEL heating. In this way, other temperature effects within the calculated $10 \mathrm{ps}$ such as hydrodynamic expansion are reduced by neglecting the PIC code particle velocities. This is justified since the cooling times for these effects are large compared to our simulation time.

The present treatment combines state-of-the-art PIC and hydrodynamic simulations in order to span several orders of magnitude in time. Although simplifying assumptions have to be made in order to convert the results, realistic density profiles throughout the sample are obtained; see Fig. 6 for the case of $10 \mathrm{ps}$.

\section{Thomson scattering signal for the full pump-probe scenario}

We have calculated the Thomson scattering spectrum according to Sec. III B for the density profiles derived from the HELIOS simulations as shown in Fig. 6, and the temperature profiles of the PIC simulation increased by $10 \%$ throughout the target (see Fig. 3). We show the respective results for different time delays between the optical laser and the FLASH probe pulse in Fig. 7 and compare with the ideal probe as displayed in Fig. 5. Most interestingly, the calcu- 


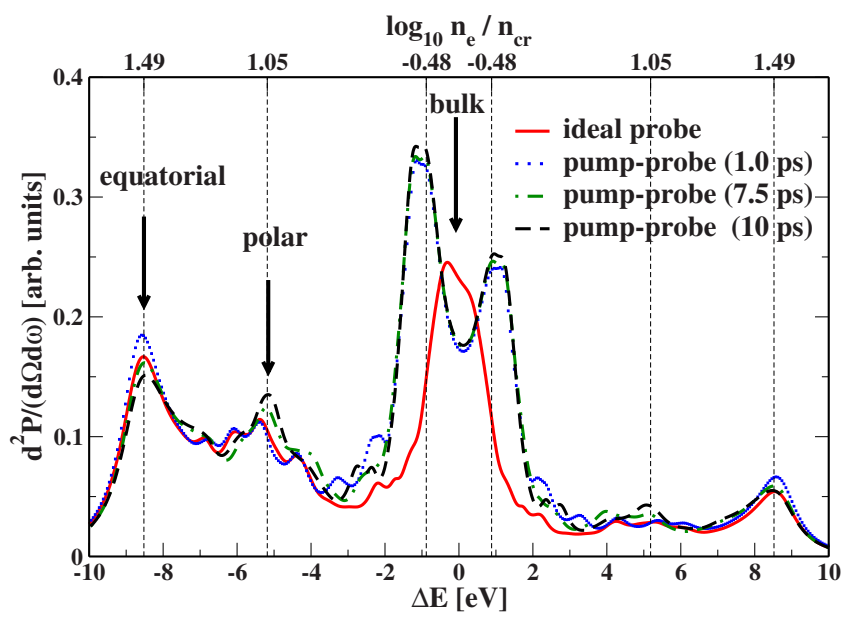

FIG. 7. (Color online) Free-electron Thomson scattering spectrum off liquid hydrogen droplets as a function of the photon energy shift $\Delta E$. The ideal probe (solid red curve; see Fig. 5) is compared with the full pump-probe scenario considering delays of 1 ps (dotted blue line), 7.5 ps (dashed-dotted green line), and 10 ps (dashed black line). The electron densities for the different maxima are given in units of the critical density of optical laser in the upper legend $\left(n_{c r}=1.7 \times 10^{21} \mathrm{~cm}^{-3}\right)$.

lated Thomson scattering spectrum allows us to monitor the temporal evolution of the electron density and temperature in the target. The radiative hydrodynamic simulations indicate that the sharp density and temperature profiles imposed by the optical laser start to dissolve in the subsequent $10 \mathrm{ps}$ considered here, especially their steep flanks, while the peak values of density and temperature are still preserved. Thus, the intensity of the equatorial plasmon (redshifted and blueshifted peaks) is decreasing. Its location however is only weakly shifted, and an effective electron density of $n_{e}=5.2$ $\times 10^{22} \mathrm{~cm}^{-3}$ and an effective electron temperature of $T_{e}$ $=8.4 \mathrm{eV}$ can be derived for $10 \mathrm{ps}$.

On the contrary, the intensity of the plasmon peak allocated to the polar region is enhanced with time. This can be attributed to scattering off the broadened flanks of the equatorial density distribution which are contributing, at least for the considered time delay of $10 \mathrm{ps,} \mathrm{just} \mathrm{in} \mathrm{the} \mathrm{density} \mathrm{and}$ temperature domains originally characteristic of the polar region alone. This "polar" peak moves slightly toward lower densities. We can derive an effective electron density of $n_{e}$ $=1.9 \times 10^{22} \mathrm{~cm}^{-3}$ and a slightly increased effective electron temperature of $T_{e}=4.4 \mathrm{eV}$.

Significant modifications in the scattering signal are to be seen in the central peak of the spectrum which stems from the bulk of the target. The increasing electron density in this region as obtained from the HELIOS simulations is due not only to additional absorption processes of FLASH photons (see Fig. 6) but also to the dissolution of the strong density gradients in the equatorial and polar regions with time. This results in a switching between noncollective scattering for ideal probing and collective scattering for the realistic case; an effective electron density of $n_{e}=6.4 \times 10^{20} \mathrm{~cm}^{-3}$ and an effective electron temperature of $T_{e}=3.2 \mathrm{eV}$ can be derived for $10 \mathrm{ps}$.

For longer time delays than 10 ps we expect a further equalization of the electron-density and temperature profiles, so that scattering contributions from different target regions mix, i.e., a mapping of target regions to features in the scattering spectrum is increasingly hindered. Furthermore, electron-ion collisions will lead to an equilibration of the electron and ion temperatures on these time scales. This relaxation process can be studied by considering the ion feature in Eq. (2) in addition to the contribution of free electrons. Hydrodynamic expansion of the droplet will occur on nanosecond time scales.

\section{CONCLUSIONS}

In this paper we have calculated the Thomson scattering signal of strongly inhomogeneous plasmas generated by an optical and probed with a soft X-ray laser. We have employed PIC simulations to derive electron-density and temperature distributions in a liquid hydrogen droplet that is pumped by an optical laser. The interaction of the FLASH probe pulse with the preheated inhomogeneous target was considered by performing radiative hydrodynamic simulations using the HELIOS code. The DSF was then calculated for each cell using a quantum statistical approach (BMA) that accounts for collisions in the plasma.

In the case studied here, i.e., an optical pump—soft x-ray probe experiment on liquid hydrogen droplets-two different pairs of plasmon peaks are seen in the Thomson scattering spectrum (see Fig. 7), which allow us to identify the plasma parameters in the equatorial and polar regions of the droplet on ultrashort time scales up to $1 \mathrm{ps}$. The density can be derived from the plasmon position and the temperature from the asymmetry of the peaks. Considering time delays between the optical laser and FLASH up to $10 \mathrm{ps,} \mathrm{the} \mathrm{changes}$ in the scattering spectrum can be mapped onto the evolution of the electron density and temperature in the target. However, a mixing of scattering contributions that stem from different target regions occurs, so that a clear identification at least of the polar region is hindered for longer time delays. The central scattering peak that stems from the bulk region switches from noncollective mode-if an ideal probe pulse is assumed-to collective mode for a realistic treatment of the FLASH probe pulse.

Our calculations show that Thomson scattering is a promising tool to diagnose inhomogeneous plasmas and to monitor their time evolution. A variation of the laser parameters, e.g., energy of the pump and the probe pulses, length of the pump and probe pulses, and delay between pump and probe, would give access to a broad region of plasma densities and temperatures of interest. The detailed analysis of this parameter space has still to be done and is subject of future work. For this purpose, efficient radiation-hydrodynamic codes with better spatial resolution than the one-dimensionalHELIOS code that was used in the present study are highly desirable. Furthermore, a proper inclusion of collisions together with collisional ionization is planned within the PIC simulations. This would give us a more detailed insight into the physics of the laser-plasma interaction on ultrashort time scales, in particular with respect to absorption processes for which different predictions exist $[43,44]$. Finally, improved theoretical approaches to the DSF including, e.g., local-field 
corrections [45] should be considered. Electron-ion relaxation processes could also be studied via the full Thomson scattering spectrum by measuring the intensity ratio of the electron and ion features separately and time resolved (see $[46,47])$.

\section{ACKNOWLEDGMENTS}

We thank E. Förster, G. Gregori, H. Reinholz, G. Röpke, and U. Zastrau for helpful discussions. This work was supported by the DFG within the SFB 652 "Strong correlations and collective effects in radiation fields: Coulomb systems, clusters, and particles" and the Federal Ministry for Education and Science (BMBF) under Grant No. FSP 301FLASH and Project No. 05KS7HRA. The work by S.H.G. was performed under the auspices of the Department of Energy by Lawrence Livermore National Laboratory under Contract No. DE-AC52-07NA27344. M.C. and C.F. acknowledge support by the Alexander von HumboldtFoundation. R.R.F. acknowledges support by GrK 1355.
[1] S. H. Glenzer and R. Redmer, Rev. Mod. Phys. 81, 1625 (2009).

[2] S. H. Glenzer et al., Phys. Rev. Lett. 82, 97 (1999).

[3] G. Gregori et al., J. Quant. Spectrosc. Radiat. Transf. 99, 225 (2006).

[4] R. W. Lee et al., Laser Part. Beams 20, 527 (2002).

[5] R. W. Lee et al., J. Opt. Soc. Am. B 20, 770 (2003).

[6] S. H. Glenzer, G. Gregori, R. W. Lee, F. J. Rogers, S. W. Pollaine, and O. L. Landen, Phys. Rev. Lett. 90, 175002 (2003).

[7] G. Gregori, S. H. Glenzer, W. Rozmus, R. W. Lee, and O. L. Landen, Phys. Rev. E 67, 026412 (2003).

[8] S. H. Glenzer et al., Phys. Rev. Lett. 98, 065002 (2007).

[9] S. H. Glenzer et al., J. Phys.: Conf. Ser. 112, 032071 (2008).

[10] H. J. Lee et al., Phys. Rev. Lett. 102, 115001 (2009).

[11] A. L. Kritcher et al., Science 322, 69 (2008).

[12] E. García Saiz et al., Nat. Phys. 4, 940 (2008).

[13] S. Glenzer et al., High Energy Density Phys. 6, 1 (2010).

[14] Relativistic Nonlinear Electrodynamics: Interaction of Charged Particles with Strong and Super Strong Laser Fields, edited by H. Avetissian (Springer, Berlin, 2006).

[15] O. L. Landen, S. H. Glenzer, M. J. Edwards, R. W. Lee, G. W. Collins, R. C. Cauble, W. W. Hsing, and B. A. Hammel, J. Quant. Spectrosc. Radiat. Transf. 71, 465 (2001).

[16] W. Ackermann et al., Nat. Photonics 1, 336 (2007).

[17] Y. Ding et al., Phys. Rev. Lett. 102, 254801 (2009).

[18] The European X-Ray Free-Electron Laser-Technical Design Report, edited by M. Altarelli et al. (DESY, Hamburg, 2007).

[19] A. Höll et al., High Energy Density Phys. 3, 120 (2007).

[20] U. Zastrau et al., Phys. Rev. E 78, 066406 (2008).

[21] B. Nagler et al., Nat. Phys. 5, 693 (2009).

[22] H. A. Baldis, J. Dunn, M. E. Foord, and W. Rozmus, Rev. Sci. Instrum. 73, 4223 (2002).

[23] R. R. Fäustlin et al., Phys. Rev. Lett. 104, 125002 (2010).

[24] C. Fortmann, R. Thiele, R. Fäustlin, T. Bornath, B. Holst, W.-D. Kraeft, V. Schwarz, S. Toleikis, T. Tschentscher, and R. Redmer, High Energy Density Phys. 5, 208 (2009).

[25] J. Chihara, J. Phys.: Condens. Matter 12, 231 (2000).

[26] J. Chihara, J. Phys. F: Met. Phys. 17, 295 (1987).
[27] G. Gregori, A. Ravasio, A. Höll, S. H. Glenzer, and S. J. Rose, High Energy Density Phys. 3, 99 (2007).

[28] S. Sahoo, G. F. Gribakin, G. Shabbir Naz, J. Kohanoff, and D Riley, Phys. Rev. E 77, 046402 (2008).

[29] E. E. Salpeter, Phys. Rev. 120, 1528 (1960).

[30] R. Thiele, T. Bornath, C. Fortmann, A. Höll, R. Redmer, H. Reinholz, G. Röpke, A. Wierling, S. H. Glenzer, and G. Gregori, Phys. Rev. E 78, 026411 (2008).

[31] N. D. Mermin, Phys. Rev. B 1, 2362 (1970).

[32] A. Selchow, G. Röpke, A. Wierling, H. Reinholz, T. Pschiwul, and G. Zwicknagel, Phys. Rev. E 64, 056410 (2001).

[33] H. Reinholz, R. Redmer, G. Röpke, and A. Wierling, Phys. Rev. E 62, 5648 (2000).

[34] R. Thiele, R. Redmer, H. Reinholz, and G. Röpke, J. Phys. A 39, 4365 (2006).

[35] A. Höll, R. Redmer, G. Röpke, and H. Reinholz, Eur. Phys. J. D 29, 159 (2004).

[36] R. Redmer, H. Reinholz, G. Röpke, R. Thiele, and A. Höll, IEEE Trans. Plasma Sci. 33, 77 (2005).

[37] C. Fortmann, T. Bornath, R. Redmer, H. Reinholz, G. Röpke, V. Schwarz, and R. Thiele, Laser Part. Beams 27, 311 (2009).

[38] A. Pukhov, J. Plasma Phys. 61, 425 (1999).

[39] A. Karmakar and A. Pukhov, Laser Part. Beams 25, 371 (2007).

[40] J. MacFarlane, I. Golovkin, and P. Woodruff, J. Quant. Spectrosc. Radiat. Transf. 99, 381 (2006).

[41] S. Kuhlbrodt, B. Holst, and R. Redmer, Contrib. Plasma Phys. 45, 73 (2005).

[42] M. R. Zaghloul, M. A. Bourham, and J. M. Doster, J. Phys. D: Appl. Phys. 34, 772 (2001).

[43] P. Mulser, H. Ruhl, and J. Steinmetz, Laser Part. Beams 19, 23 (2001).

[44] A. V. Brantov, V. Y. Bychenkov, W. Rozmus, C. E. Capjack, and R. Sydora, Phys. Plasmas 10, 4633 (2003).

[45] C. Fortmann, A. Wierling, and G. Röpke, Phys. Rev. E 81, 026405 (2010).

[46] G. Gregori and D. O. Gericke, EPL 83, 15002 (2008).

[47] G. Gregori and D. O. Gericke, Phys. Plasmas 16, 056306 (2009). 